\title{
Consejo de Seguridad
}

Distr. general

8 de noviembre de 2002

Español

Original: inglés

\section{Resolución 1441 (2002)}

\section{Aprobada por el Consejo de Seguridad en su $4644^{\text {a }}$ sesión, celebrada el 8 de noviembre de 2002}

\section{El Consejo de Seguridad,}

Recordando todas sus resoluciones anteriores en la materia, en particular sus resoluciones 661 (1990), de 6 de agosto de 1990, 678 (1990), de 29 de noviembre de 1990, 686 (1991), de 2 de marzo de 1991, 687 (1991), de 3 de abril de 1991, 688 (1991), de 5 de abril de 1991, 707 (1991), de 15 de agosto de 1991, 715 (1991), de 11 de octubre de 1991, 986 (1995), de 14 de abril de 1995, y 1284 (1999), de 17 de diciembre de 1999, así como todas las declaraciones de su Presidencia sobre la cuestión,

Recordando también su resolución 1382 (2001), de 29 de noviembre de 2001, y su intención de aplicarla plenamente,

Reconociendo la amenaza que el incumplimiento por el Iraq de las resoluciones del Consejo y la proliferación de armas de destrucción en masa y misiles de gran alcance plantean para la paz y la seguridad internacionales,

Recordando que en su resolución 678 (1990) autorizó a los Estados Miembros a que utilizaran todos los medios necesarios para hacer valer y llevar a la práctica la resolución 660 (1990), de 2 de agosto de 1990, y todas las resoluciones pertinentes aprobadas ulteriormente y para restablecer la paz y la seguridad internacionales en la región,

Recordando además que en la resolución 687 (1991) se imponían obligaciones al Iraq como paso necesario para cumplir su objetivo declarado de restablecer la paz y la seguridad internacionales en la región,

Deplorando que el Iraq no haya hecho una declaración exacta, cabal, definitiva y completa, como se exigía en la resolución 687 (1991), de todos los aspectos de sus programas de desarrollo de armas de destrucción en masa y misiles balísticos con un alcance de más de ciento cincuenta kilómetros ni de las armas de esa índole que tuviera en su poder, sus componentes e instalaciones y lugares de producción, así como de todos los demás programas nucleares, incluidos aquellos que, según afirme, obedecen a fines no relacionados con material utilizable para armas nucleares,

Deplorando además que el Iraq haya obstruido reiteradamente el acceso inmediato, incondicional e irrestricto a sitios designados por la Comisión Especial de las 
Naciones Unidas (UNSCOM) y el Organismo Internacional de Energía Atómica (OIEA), no haya cooperado plena e incondicionalmente con los inspectores de la UNSCOM y el OIEA, como se exigía en la resolución 687 (1991), y finalmente haya puesto término en 1998 a todo tipo de cooperación con la UNSCOM y el OIEA,

Deplorando que, desde diciembre de 1998, no haya habido en el Iraq ninguna forma de vigilancia, inspección y verificación, como requerían las resoluciones pertinentes, de las armas de destrucción en masa y misiles balísticos, a pesar de las repetidas exigencias del Consejo al efecto de que el Iraq proporcionara acceso inmediato, incondicional e irrestricto a la Comisión de las Naciones Unidas de Vigilancia, Verificación e Inspección (UNMOVIC), establecida en la resolución 1284 (1999) como organización sucesora de la UNSCOM, ni al OIEA, y deplorando la consiguiente prolongación de la crisis en la región y los sufrimientos del pueblo iraquí,

Deplorando también que el Gobierno del Iraq no haya cumplido los compromisos que contrajo en virtud de la resolución 687 (1991) con respecto al terrorismo, en virtud de la resolución 688 (1991) de poner fin a la represión de su población civil y dar acceso a las organizaciones humanitarias internacionales a todos los que necesitaran asistencia en el Iraq, y, en virtud de las resoluciones 686 (1991), 687 (1991) y 1284 (1999) de devolver a los nacionales de Kuwait y de terceros países que tenía detenidos ilícitamente o devolver bienes de propiedad de Kuwait de los que se había incautado ilícitamente o de cooperar para determinar su paradero,

Recordando que en su resolución 687 (1991) había declarado que una cesación del fuego estaría subordinada a que el Iraq aceptara las disposiciones de esa resolución, incluidas las obligaciones de su cargo que en ella figuraban,

Decidido a lograr que el Iraq cumpla plena e inmediatamente y sin condiciones ni restricciones las obligaciones que le imponen la resolución 687 (1991) y otras resoluciones en la materia y recordando que las resoluciones del Consejo constituyen la norma para determinar el cumplimiento por parte del Iraq,

Recordando que el funcionamiento efectivo de la UNMOVIC, en su calidad de organización sucesora de la Comisión Especial, y del OIEA es esencial para la aplicación de la resolución 687 (1991) y otras resoluciones en la materia,

Tomando nota de que la carta que, con fecha 16 de septiembre de 2002, dirigió al Secretario General el Ministro de Relaciones Exteriores del Iraq constituye un primer paso necesario para rectificar el persistente incumplimiento por el Iraq de las resoluciones del Consejo en la materia,

Tomando nota además de la carta que, con fecha 8 de octubre de 2002, dirigió al Presidente Ejecutivo de la UNMOVIC y al Director General del OIEA el General Al-Saadi del Gobierno del Iraq, en que se enunciaban los arreglos prácticos formulados a raíz de su reunión en Viena, que constituyen requisitos previos para que la UNMOVIC y el OIEA reanuden las inspecciones en el Iraq, y expresando su más profunda preocupación por el hecho de que el Gobierno del Iraq siga sin confirmar los arreglos indicados en esa carta,

Reafirmando el compromiso de todos los Estados Miembros con la soberanía y la integridad territorial del Iraq, Kuwait y los Estados vecinos, 
Encomiando al Secretario General y a los miembros de la Liga de los Estados Árabes y su Secretario General por sus gestiones a este respecto,

Decidido a lograr que se cumplan plenamente sus decisiones,

Actuando en virtud del Capítulo VII de la Carta de las Naciones Unidas,

1. Decide que el Iraq ha incurrido y sigue incurriendo en violación grave de sus obligaciones con arreglo a las resoluciones en la materia, entre ellas la resolución 687 (1991), en particular al no cooperar con los inspectores de las Naciones Unidas y con el OIEA y no llevar a cabo las medidas previstas en los párrafos 8 a 13 de la resolución 687 (1991);

2. Decide, al tiempo que reconoce lo indicado en el párrafo 1 supra, conceder al Iraq, en virtud de la presente resolución, una última oportunidad de cumplir sus obligaciones en materia de desarme con arreglo a las resoluciones pertinentes del Consejo; y decide en consecuencia instaurar un régimen de inspección reforzado con el objetivo de llevar a una conclusión cabal y verificada el proceso de desarme establecido por la resolución 687 (1991) y las resoluciones ulteriores del Consejo;

3. Decide que, a fin de comenzar a cumplir sus obligaciones en materia de desarme, además de presentar las declaraciones semestrales requeridas, el Gobierno del Iraq deberá proporcionar a la UNMOVIC, el OIEA y el Consejo, dentro de los treinta días siguientes a la fecha de la presente resolución, una declaración que a esa fecha sea exacta, cabal y completa de todos los aspectos de sus programas para el desarrollo de armas químicas, biológicas y nucleares, misiles balísticos y otros sistemas vectores como vehículos aéreos no tripulados y sistemas de dispersión diseñados para ser utilizados en aeronaves, incluidas todas las existencias y ubicaciones precisas de este tipo de armas, componentes, subcomponentes, reservas de agentes, y del material y equipo conexo, de las ubicaciones y la labor de sus instalaciones de investigación, desarrollo y producción, así como de todos los demás programas químicos, biológicos y nucleares, incluidos aquellos que, según afirme, obedecen a fines no relacionados con material para armamentos o la producción de armamentos;

4. Decide que las falsedades u omisiones en las declaraciones presentadas por el Iraq en cumplimiento de la presente resolución y el hecho de que el Iraq deje en cualquier momento de cumplir la presente resolución y de cooperar plenamente en su aplicación constituirán una nueva violación grave de las obligaciones del Iraq y se comunicarán al Consejo para su evaluación de conformidad con los párrafos 11 y 12 infra;

5. Decide que el Iraq deberá proporcionar a la UNMOVIC y al OIEA acceso inmediato, sin trabas, incondicional e irrestricto a todas y cada una de las zonas, incluidas las subterráneas, instalaciones, edificios, equipo, registros y medios de transporte que deseen inspeccionar, así como acceso inmediato, sin trabas, irrestricto y privado a todos los funcionarios y otras personas a quienes la UNMOVIC o el OIEA deseen entrevistar en la forma o el lugar que decidan la UNMOVIC o el OIEA en relación con cualquier aspecto de sus mandatos; decide además que la UNMOVIC y el OIEA, ejerciendo su discreción, podrán realizar entrevistas dentro o fuera del Iraq y podrán facilitar el traslado de las personas entrevistadas y de sus familiares fuera del Iraq y que queda librado exclusivamente a la discreción de la UNMOVIC y el OIEA hacer que esas entrevistas tengan lugar sin que estén presentes observadores del Gobierno del Iraq; y encomienda a la UNMOVIC y pide al OIEA aue reanuden las insneccinnes dentro de los marenta y cinco días cimientes 
a la aprobación de la presente resolución y que le pongan al corriente dentro de los sesenta días siguientes a esa fecha;

6. Hace suya la carta de fecha 8 de octubre de 2002 dirigida al General Al-Saadi del Gobierno del Iraq por el Presidente Ejecutivo de la UNMOVIC y el Director General del OIEA, que se adjunta como anexo a la presente resolución, y decide que lo indicado en la carta tendrá carácter obligatorio para el Iraq;

7. Decide además, habida cuenta de la prolongada interrupción por el Iraq de la presencia de la UNMOVIC y del OIEA y de manera que éstos puedan cumplir las tareas estipuladas en la presente resolución y todas las resoluciones anteriores en la materia, y no obstante los entendimientos anteriores, establecer por la presente las siguientes disposiciones revisadas o adicionales, que serán obligatorias para el Iraq, para facilitar su labor en el Iraq:

- La UNMOVIC y el OIEA determinarán la composición de sus equipos de inspección y se asegurarán de que estén integrados por los expertos más calificados y experimentados de que se disponga;

- Todo el personal de la UNMOVIC y el OIEA gozará de las prerrogativas e inmunidades correspondientes a las de los expertos en misión contempladas en la Convención sobre Prerrogativas e Inmunidades de las Naciones Unidas y el Acuerdo sobre los privilegios e inmunidades del OIEA;

- La UNMOVIC y el OIEA tendrán derecho irrestricto de entrada y salida del Iraq y derecho de circulación sin trabas, irrestricta e inmediata de ida y vuelta a los sitios de inspección, y derecho a inspeccionar cualquier sitio y edificio, incluido el acceso inmediato, sin trabas, incondicional e irrestricto a los sitios presidenciales en condiciones iguales a las de otros sitios, no obstante lo dispuesto en la resolución 1154 (1998) de 2 de marzo de 1998;

- La UNMOVIC y el OIEA tendrán derecho a obtener del Iraq los nombres de todo el personal que esté o haya estado relacionado con los programas químicos, biológicos, nucleares y de misiles balísticos del Iraq y las instalaciones de investigación, desarrollo y producción conexas;

- La protección de las instalaciones de la UNMOVIC y el OIEA estará a cargo de un número suficiente de guardias de seguridad de las Naciones Unidas;

- La UNMOVIC y el OIEA, a los efectos de impedir los movimientos en los sitios que deban ser inspeccionados, tendrán derecho a declarar zonas de exclusión, que pueden comprender las zonas circundantes y corredores de tránsito, en las que el Iraq suspenderá el tránsito terrestre y aéreo de forma que en un sitio que se esté inspeccionando no se cambie ni se saque nada;

- La UNMOVIC y el OIEA tendrán derecho libre e irrestricto a utilizar y hacer aterrizar aviones y helicópteros, incluidos vehículos de reconocimiento tripulados y no tripulados;

- La UNMOVIC y el OIEA tendrán derecho, librado a su exclusiva discreción, a retirar, destruir o inutilizar de manera verificable todas las armas, subsistemas, componentes, registros, materiales y otros artículos conexos prohibidos, y derecho a requisar o clausurar cualesquiera instalaciones o equipo para su producción; y 
- La UNMOVIC y el OIEA tendrán derecho a importar y utilizar libremente equipo o materiales para las inspecciones y a requisar y exportar cualquier equipo, materiales o documentos obtenidos durante las inspecciones, sin que pueda registrarse al personal de la UNMOVIC y el OIEA o su equipaje oficial y personal;

8. Decide asimismo que el Iraq no realizará ni amenazará con realizar actos hostiles contra ningún representante o miembro del personal de las Naciones Unidas o del OIEA o de cualquier Estado Miembro que adopte medidas para hacer cumplir cualquiera de sus resoluciones;

9. Pide al Secretario General que notifique inmediatamente al Iraq la presente resolución, que tiene fuerza obligatoria para el Iraq; exige que el Iraq confirme en un plazo de siete días a partir de dicha notificación su intención de cumplir plenamente la presente resolución; y exige además que el Iraq coopere inmediata, incondicional y activamente con la UNMOVIC y el OIEA;

10. Pide a todos los Estados Miembros que presten pleno apoyo a la UNMOVIC y al OIEA en el cumplimiento de sus mandatos, incluso facilitando cualquier información relacionada con programas prohibidos $u$ otros aspectos de sus mandatos, incluidos los intentos hechos por el Iraq desde 1998 para adquirir artículos prohibidos y recomendando sitios que puedan inspeccionarse, personas que puedan entrevistarse, las condiciones de esas entrevistas y los datos que sea necesario reunir, y que la UNMOVIC y el OIEA le comuniquen los resultados de todo ello;

11. Encomienda al Presidente Ejecutivo de la UNMOVIC y al Director General del OIEA que le informen inmediatamente de toda injerencia del Iraq en las actividades de inspección, así como de todo incumplimiento por el Iraq de sus obligaciones en materia de desarme, incluidas sus obligaciones relativas a las inspecciones en virtud de la presente resolución;

12. Decide reunirse inmediatamente una vez recibido un informe presentado de conformidad con los párrafos 4 u 11 supra a fin de examinar la situación y la necesidad de que se cumplan plenamente todas sus resoluciones en la materia con objeto de asegurar la paz y la seguridad internacionales;

13. Recuerda, en este contexto, que ha advertido reiteradamente al Iraq que, de seguir infringiendo sus obligaciones, se expondrá a graves consecuencias;

14. Decide seguir ocupándose de la cuestión. 
Texto de la carta Blix/EIBaradei

\title{
Comisión de las Naciones Unidas de Vigilancia, Verificación e Inspección
}

\author{
EI Presidente Ejecutivo
}

\section{Organismo Internacional de Energía Atómica}

\section{El Director General}

8 de octubre de 2002

Estimado General Al-Saadi:

En el curso de nuestra reunión celebrada en Viena debatimos arreglos prácticos que constituían requisitos previos para que la UNMOVIC y el OIEA reanudaran las inspecciones en el Iraq. Como recordará usted, al concluir la reunión de Viena acordamos una declaración que enumeraba algunos de los principales resultados logrados, en particular la aceptación por el Iraq de todos los derechos de inspección contemplados en todas las resoluciones del Consejo de Seguridad en la materia. Se indicó que esa aceptación se producía sin condiciones.

En la sesión de 3 de octubre de 2002 en que informamos al Consejo de Seguridad, los miembros del Consejo sugirieron que preparáramos un documento sobre todas las conclusiones que habíamos alcanzado en Viena. En la presente carta se enumeran esas conclusiones y se recaba su confirmación de ellas. Informaremos en consecuencia al Consejo de Seguridad.

En la declaración hecha al término de la reunión se aclaró que se otorgará a la UNMOVIC y al OIEA acceso inmediato, incondicional e irrestricto a los sitios, incluidos aquellos que anteriormente habían sido calificados de "sitios sensibles". No obstante, como se observó, ocho sitios presidenciales han sido objeto de procedimientos especiales en virtud de un memorando de entendimiento de 1998. Si hubiera que otorgar acceso inmediato, incondicional e irrestricto a esos sitios, como a todos los demás sitios, la UNMOVIC y el OIEA llevarían a cabo sus inspecciones en ellos con la misma profesionalidad.

Confirmamos nuestro entendimiento de que la UNMOVIC y el OIEA tienen derecho a determinar el número de inspectores que se precisará para acceder a cada sitio de inspección. Esa decisión se tomará sobre la base del tamaño y la complejidad del sitio. Confirmamos también que se informará al Iraq en caso de que se designen sitios adicionales, es decir, sitios que no hayan sido declarados por el Iraq o que no hayan sido inspeccionados anteriormente por la Comisión Especial de las Naciones Unidas (UNSCOM) o el OIEA, mediante una Notificación de Inspección que se presentará en el momento de la llegada de los inspectores a esos sitios.

Excelentísimo Señor

General Amir H. Al-Saadi

Asesor

Oficina Presidencial

Bagdad

Irad 
El Iraq ha de asegurar que no se destruirá ningún material o equipo prohibidos, registros ni otros artículos pertinentes salvo en presencia de los inspectores de la UNMOVIC o del OIEA, según proceda, y a petición de éstos.

La UNMOVIC y el OIEA podrán entrevistar a cualquier persona en el Iraq que consideren que podría tener información pertinente a su mandato. El Iraq deberá facilitar esas entrevistas y la UNMOVIC y el OIEA elegirán el modo y el lugar en que éstas se llevarán a cabo.

La Dirección Nacional de Vigilancia será, como anteriormente, el interlocutor iraquí de los inspectores. El Centro de Vigilancia y Verificación Permanentes de Bagdad (BOMVIC) se mantendrá en el mismo lugar y en las mismas condiciones que el antiguo Centro de Vigilancia y Verificación de Bagdad. La Dirección Nacional de Vigilancia prestará servicios gratuitos, como anteriormente, para el acondicionamiento de los locales.

La Dirección Nacional de Vigilancia proporcionará de forma gratuita: a) acompañantes para facilitar el acceso a los sitios de inspección y la comunicación con el personal que vaya a ser entrevistado; b) una línea de comunicación directa para el BOMVIC que será atendida las 24 horas del día y los siete días de la semana por una persona que hable inglés; c) el apoyo que se solicite en cuanto a personal y transporte terrestre dentro del país; y d) asistencia en el transporte de material y equipo, a petición de los inspectores (equipo de construcción, excavación, etc.). La Dirección Nacional de Vigilancia también pondrá acompañantes a disposición de los inspectores en caso de que se realicen inspecciones fuera del horario normal de trabajo, incluso de noche y en días feriados.

Podrán establecerse oficinas regionales de la UNMOVIC y el OIEA, por ejemplo en Basra y Mosul, para uso de sus respectivos inspectores. Para este fin, el Iraq proporcionará de forma gratuita los edificios de oficinas necesarios, alojamiento para el personal y los acompañantes que se necesiten.

La UNMOVIC y el OIEA podrán usar cualquier sistema de transmisión de voz o datos, incluso redes internas o por satélite, con o sin capacidad de cifrado. La UNMOVIC y el OIEA también podrán instalar equipos sobre el terreno con capacidad de transmisión directa de datos al BOMVIC, Nueva York y Viena (por ejemplo, sensores y cámaras de vigilancia). El Iraq facilitará esta actividad y no interferirá con las comunicaciones de la UNMOVIC ni del OIEA.

El Iraq proporcionará de forma gratuita protección física para todo el equipo de vigilancia y construirá antenas de transmisión remota de datos, a petición de la UNMOVIC y del OIEA. A petición de la UNMOVIC, por conducto de la Dirección Nacional de Vigilancia, el Iraq asignará frecuencias para el equipo de comunicaciones.

El Iraq prestará servicios de seguridad a todo el personal de la UNMOVIC y el OIEA. Designará lugares de alojamiento seguros y adecuados, a unas tarifas normales, para ese personal. Por su parte, la UNMOVIC y el OIEA exigirán a su personal que se aloje única y exclusivamente en los lugares concertados con el Iraq.

En cuanto al uso de aviones para el transporte de personal y equipos y para fines de inspección, se aclaró que los aviones utilizados por el personal de la UNMOVIC y el OIEA podrán aterrizar en el aeropuerto internacional Saddam a su llegada a Bagdad. Los puntos de partida de los aviones los decidirá la UNMOVIC. La base aérea Rasheed seguirá utilizándose para las operaciones con helicóptero de la 
UNMOVIC y el OIEA. La UNMOVIC y el Iraq establecerán oficinas de enlace aéreo en su base aérea. Tanto en el aeropuerto internacional Saddam como en la base aérea Rasheed, el Iraq proporcionará los recintos e instalaciones de apoyo necesarios. El Iraq proporcionará combustible gratis para los aviones, como lo ha hecho anteriormente.

Con respecto al tema más amplio de las operaciones aéreas en el Iraq, tanto con aviones como con helicópteros, el Iraq garantizará la seguridad de éstas en su espacio aéreo fuera de las zonas de prohibición de vuelos. En cuanto a las operaciones aéreas en las zonas de prohibición de vuelos, el Iraq tomará todas las medidas que pueda para garantizar la seguridad de esas operaciones.

Se podrán utilizar helicópteros, según sea necesario, durante las inspecciones y para realizar actividades técnicas, como la detección de rayos gamma, en todo el Iraq, sin limitaciones y sin exceptuar ninguna zona. También se podrán usar helicópteros para evacuaciones por motivos médicos.

En cuanto a la cuestión de las imágenes aéreas, la UNMOVIC tal vez decida reanudar las operaciones de sobrevuelo con aviones U-2 o Mirage. Las disposiciones prácticas que se adoptarían llegado el caso serían similares a las que se han aplicado en el pasado.

Los visados para todo el personal que vaya a entrar en el país se expedirán, al igual que antes, en el puerto de entrada mediante un laissez-passer o un certificado de las Naciones Unidas; no se exigirá ningún otro requisito para entrar o salir del país. La lista de pasajeros de cada avión se proporcionará una hora antes del aterrizaje en Bagdad. No se registrará al personal de la UNMOVIC y el OIEA, ni el equipaje oficial ni personal de éste. La UNMOVIC y el OIEA se asegurarán de que su personal respeta las leyes del Iraq que restringen la exportación de determinados artículos, por ejemplo aquellos que forman parte del patrimonio cultural nacional. La UNMOVIC y el OIEA podrán llevar y traer del Iraq todos los artículos y materiales que precisen, incluidos teléfonos por satélite y otro equipo. Con respecto a las muestras, la UNMOVIC y el OIEA las repartirán, en la medida en que sea viable, de forma que el Iraq pueda recibir una porción mientras que otra se guarda como referencia. Según proceda, las organizaciones enviarán muestras a más de un laboratorio para su análisis.

Agradeceríamos que nos confirmara si lo expuesto supra refleja correctamente nuestras conversaciones de Viena.

Naturalmente, es posible que necesitemos acordar otras disposiciones prácticas una vez procedamos a realizar las inspecciones, en cuyo caso, al igual que en lo que respecta a lo mencionado más arriba, esperamos poder contar con la absoluta cooperación del Iraq.

Aprovechamos la oportunidad para saludarle muy atentamente,

(Firmado)

Hans Blix

Presidente Ejecutivo

Comisión de las Naciones Unidas de

Vigilancia, Verificación e Inspección
(Firmado)

Mohamed ElBaradei

Director General

Organismo Internacional de

Energía Atómica 